\title{
对氨基酚在二氧化钛悬浮液 中光解产生的自由基*
}

\author{
陈次平任新民 \\ (中国科学院感光化学研究所, 北京 100101 ) \\ 陆道惠徐广智** \\ (中国科学院化学研究所, 北京 100080)
}

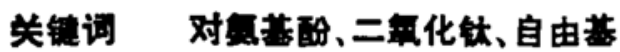

最近, 用半导体光催化分解有害物质的研究引起人们极大兴趣, 这是因为选择适当的半导 体催化剂可以利用太阳能处理毒物, 节约能源. 同时如 $\mathrm{TiO}_{2}$ 这样的半导体的光生空 穴具有 很强的氧化能力, 能分解许多有机物质 ${ }^{[1-3]}$. 而且将它们最终分解为二氧化碳、水和无机物, 避 免通常用化学方法处理废水带来的二次污染. 在毒物被氧化成最终产物的过程中, 有许多氧 化的中间物产生. 因此研究中间体的形成对了解反应机理是十分重要的.

对氨基酚是一种彩色显影剂, 它能改变人体淋巴样干细胞的 DNA 结构 ${ }^{[4]}$, 对肾脏有严重 损害曰, 是一种不可忽视的有机毒物. 因此用半导体光催化对氨基酚的分解自然会引起人们 的关注.

Bolton 等 ${ }^{\left[{ }^{6}\right.}$ 用 ESR 和自旋捕捉技术相结合的方法研究了 4-氯酚水溶液光解产生的短寿 命自由基,提出其初级自由基的产生是由 $\mathrm{C}-\mathrm{Cl}$ 键均列,尔后再引发产生次级自由基.Neta等 ${ }^{\text {[? }}$ 用 ESR 研究了对氨基酚原位辐射分解, 观察到对氨基苯氧自由基, 认为它是初级自由基· $\mathrm{OH}$ 与对氮基酚间夺氢反应给出的次级自由基. 因此研究 $\mathrm{TiO}_{2}$ 催化对氨基酚光降解的机理是属 于 Bolton 型或 Neta 型, 或是二者兼而有之, 甚至是另一种类型, 对深人研究半导体光催化 降解污物有重要意义.

\section{1 实 验 部 分}

\section{1 试剂}

5,5-二甲基-1-吡咯晽- $\mathrm{N}$-氧化物 (DMPO) 为南京大学实验化工厂生产的试剂. $\mathrm{TiO}_{2}$ 、 对氨基酚、邻氨基酚为分析纯试剂. 水为二次蒸馏水.

\section{2 仪器}

所用仪器为德国 Bruker 公司的 ESP 300 型 ESR 波谱仪. 室温下用扁平池测谱. $25 \mathrm{mg}$

1992-12-11 收稿, 1993-03-10收修改稿.

- 国家自然科学基金、北京分子动态及稳态结构化学国家实验空资助项目.

**通讯联系人. 
$\mathrm{TiO}_{2}$ 粉末加人 $5 \mathrm{ml} \mathrm{H} \mathrm{H}_{2} \mathrm{O}$ 用超声发生器进行分散. 然后在 $0.25 \mathrm{ml}$ 分散体 中加入 DMPO $\left(5 \times 10^{-2} \mathrm{~mol} / \mathrm{L}\right)$ 和对氮基酚 $\left(2 \times 10^{-3} \mathrm{~mol} / \mathrm{L}\right)$. 用 GCQ-200 型高压永灯照射至 ESR 信 号最强. 测谱前样品通高纯氮除篻. pH 值用 PHS-2 型酸度计测定.

\section{2 结 果 与 讨 论}

当对氨基酚加人含有 $\mathrm{DMPO}$ 的 $\mathrm{TiO}_{2}-\mathrm{H}_{2} \mathrm{O}$ 的悬浮液 $(\mathrm{pH}-6)$ 后, 用紫外光照射 $20 \mathrm{~min}$ 即观察到如图 1(a) 所示的 ESR 谱. 谱图颇为复杂. 欲确定这些谱线的归属, 首先 估计该体系可能的光反应过程及产生的自由基. 设想有以下反应发生:

$$
\begin{aligned}
& \mathrm{H}_{2} \mathrm{~N}-\langle\mathrm{O}\rangle \mathrm{OH} \stackrel{\mathrm{h} \nu}{\longrightarrow} \mathrm{H}_{2} \mathrm{~N}-\left\langle\mathrm{O}-\mathrm{O}^{\cdot}+\mathrm{H}^{\cdot}\right. \\
& \mathrm{H}_{2} \mathrm{~N}-\langle\mathrm{O}\rangle-\mathrm{OH} \stackrel{\mathrm{h} \nu}{\longrightarrow} \cdot\left\langle\mathrm{OH}+\cdot \mathrm{NH}_{2}\right. \\
& \mathrm{TiO}_{2} \stackrel{\mathrm{h} \nu}{\longrightarrow} \mathrm{h}^{+}+\mathrm{e}^{-} \\
& \mathrm{h}^{+}+\mathrm{H}_{2} \mathrm{O} \longrightarrow \cdot \mathrm{OH}+\mathrm{H}^{+} \\
& \mathrm{e}^{-}+\mathrm{O}_{2} \longrightarrow \mathrm{O}_{i}^{-}
\end{aligned}
$$
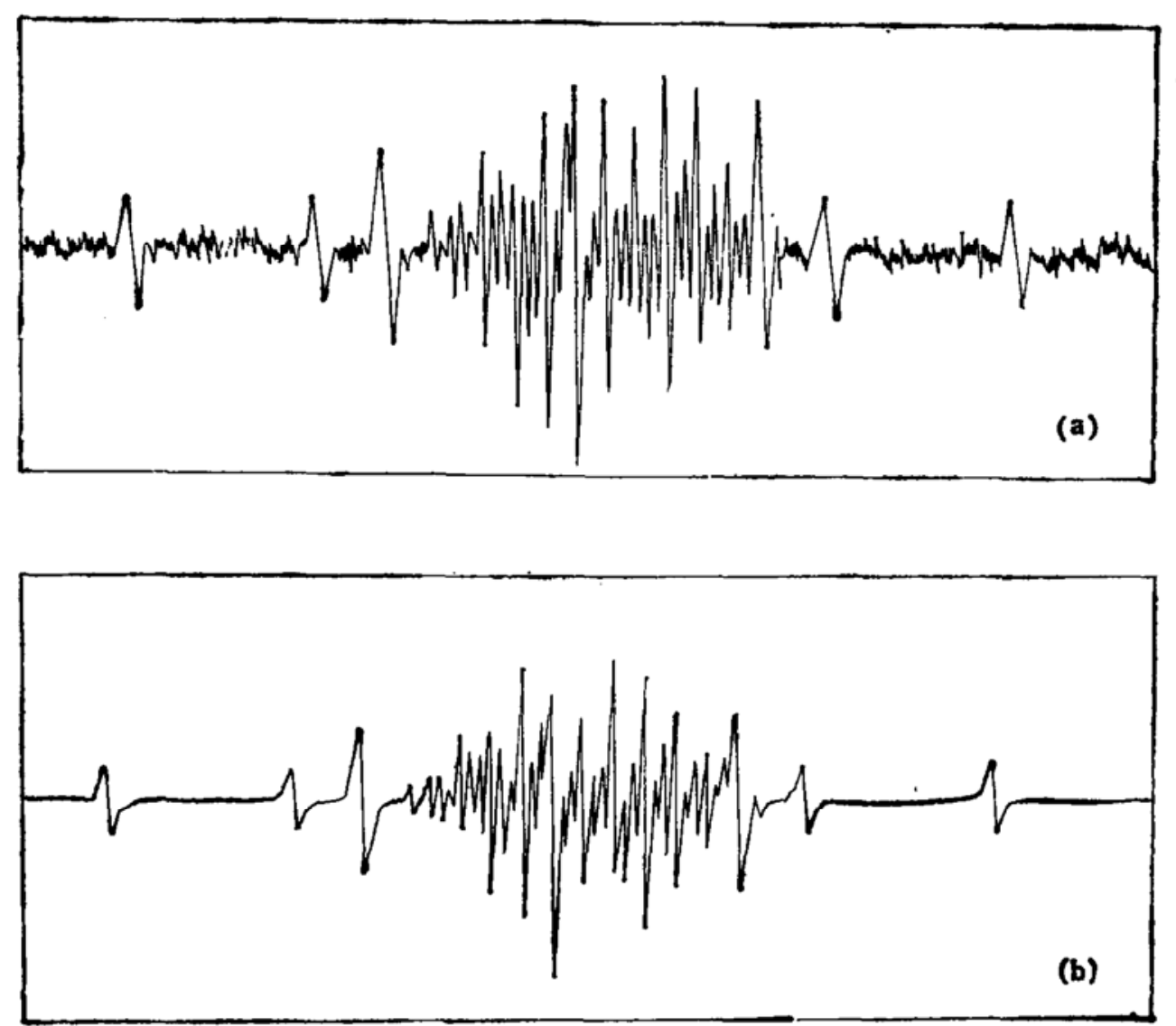

图 1

（a）对氨基酚在含 $\mathrm{DMPO}$ 的 $\mathrm{TiO}_{2}$ 暴浮液中光照得到的 $\mathrm{ESR}$ 谱图;

(b) 图 3(a):图 3(b) 按 2:1 合成得到的模拟谱 


$$
\begin{aligned}
& \mathrm{H}_{2} \mathrm{~N}-\langle\mathrm{O}\rangle-\mathrm{OH}+{ }^{\cdot} \mathrm{OH} \longrightarrow \mathrm{H}_{2} \mathrm{~N}-O \mathrm{O}-\mathrm{O}^{\cdot}+\mathrm{H}_{2} \mathrm{O} \\
& \mathrm{H}^{\cdot}+{ }^{\cdot \mathrm{OH}} \longrightarrow \mathrm{H}_{2} \mathrm{O}
\end{aligned}
$$

其次, 考虑以上反应产生的自由基与 DMPO 形成自旋 加 合物 的 ESR 特征如何. 已知 $(\mathrm{DMPO}-\mathrm{H})^{*}$ 给出三组三重峰 ${ }^{[8]}$, 分裂常数 $a_{\mathrm{N}}=1.66 \mathrm{mT}, a_{\mathrm{H}}=2.25 \mathrm{mT}$; (DMPO-OH $)^{\circ}$ 为四重峰 ${ }^{[9]}$, 分裂常数 $a_{\mathrm{N}}-a_{\mathrm{H}}=1.5 \mathrm{mT}$; (DMPO- $\left.\mathrm{NH}_{2}\right)^{\circ}$ 为 16 重峰 ${ }^{[0]}$, 分裂常数 $u_{\mathrm{N}}=$ $1.585 \mathrm{mT}, a_{\mathrm{H}}=1.903 \mathrm{mT}, a_{\mathrm{N}\left(\mathrm{NH}_{2}\right)}=0.171 \mathrm{mT} ;\left(\mathrm{DMPO}-\mathrm{O}_{2}^{-}\right)^{\circ}$ 为 12 重峰 ${ }^{[11]}$, 分裂常数 $a_{\mathrm{N}}=$ $1.41 \mathrm{mT}, a_{\mathrm{H}}^{\ominus}-1.13 \mathrm{mT}, a_{\mathrm{H}}^{r}-0.125 \mathrm{mT}$. 目前尚无 $\mathrm{DMPO}$ 与 $\mathrm{H}_{2} \mathrm{~N}-\langle\bigcirc\rangle-0^{\circ}$ 及 HO-〈O $\langle$. 形成的自旋加合物的 ESR 数据可资利用. 根据这些数据容易看出实测谱中 有 (DMPO-H $)^{*}$ 的贡献. 这可能是对氨基酚按方程 (1) 反应的结果. 为考察对氨基酚光解 反应中是否有对氨基苯氧自由基产生，我们测定了对氨基酚均相中相同浓度及光炤条件下的 ESR 谱 (图 2(a)). 由图可见在无 $\mathrm{TiO}_{2}$ 存在下得到的 ESR 谱与图 1(a) 相似, 只是不同种 类自由基信号强度比不同. 如果体系中不含 OMPO 得到了类似图 2(a) 的 ESR 谱, 只是 (OMPO-H $)^{*}$ 的三组三重峰未出现, 这是因为 $\mathrm{H}^{*}$ 寿命很短, 不能被常规的 ESR 方法检测, 该谱很可能来自对氨基酚光解产生的对氨基苯氧自由基. 当体系的 $\mathrm{pH}$ 值调至 12 时, 此自由
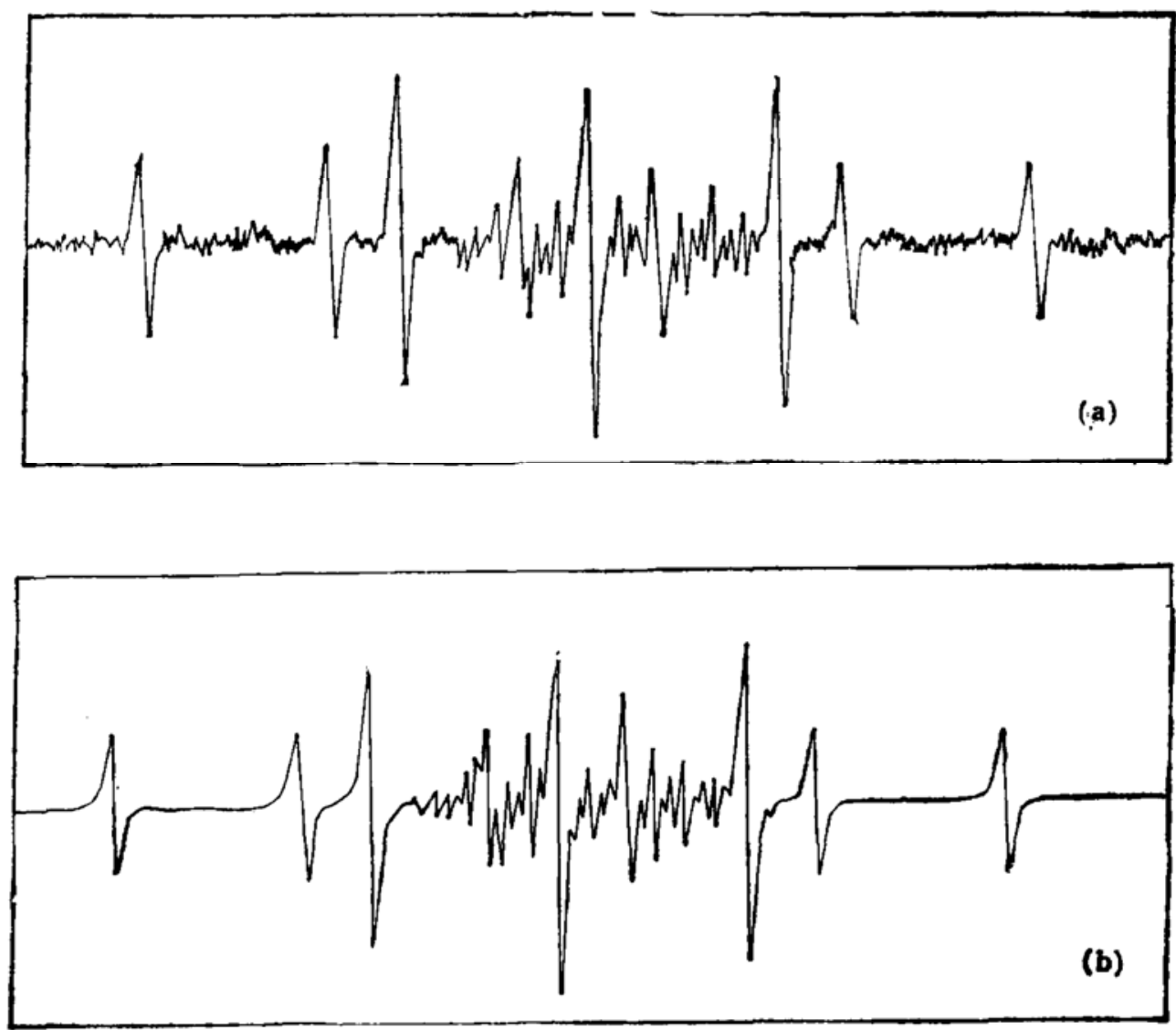

图 2

(a) 对氨基酚在含 DMPO 的水溶液中光炤得到的 ESR 谱图;

(b) 图 3(a):图 3(b) 按 0.5:1 合成得到的模拔谱 
基的 ESR 信号增强, 表明是由未偶电子与环质子及 $\mathrm{NH}_{2}$ 上质子、氮相互作用给出的 $3 \times 3 \times$ $3 \times 3-81$ 条峰, 由于谱线重叠, 实测谱只出现 33 条峰. 经计算谱与实测谱的拟合可以肯定 实测谱图 1(a) 和图 2(a) 中都含有对氨基苯氧自由基的贡献. 并且得到偶合常数 $a_{2.6}^{\mathrm{H}}=0.275$ $\mathrm{mT}, a_{3,5}^{\mathrm{H}}=0.175 \mathrm{mT}, a_{\mathrm{NH}_{2}}^{\mathrm{N}}=0.525 \mathrm{mT}, a_{\mathrm{NH}_{2}}^{\mathrm{H}}=0.550 \mathrm{mT}$. 图 3(a) 是对氨基苯氧自由基的模 拟谱, 图 3(b) 是氢原子的 DMPO 自旋加合物的模拟谱, 相应的分裂常数 $a_{\mathrm{N}}=1.647 \mathrm{mT}$, $a_{H}(2)=2.248 \mathrm{mT}$. 图 $1(\mathrm{~b})$ 及图 2(b) 分别是由 $3 \mathrm{a}: 3 \mathrm{~b}-2: 1$ 及 $3 \mathrm{a}: 3 \mathrm{~b}-0.5: 1$ 合成得到的 模拟谱.
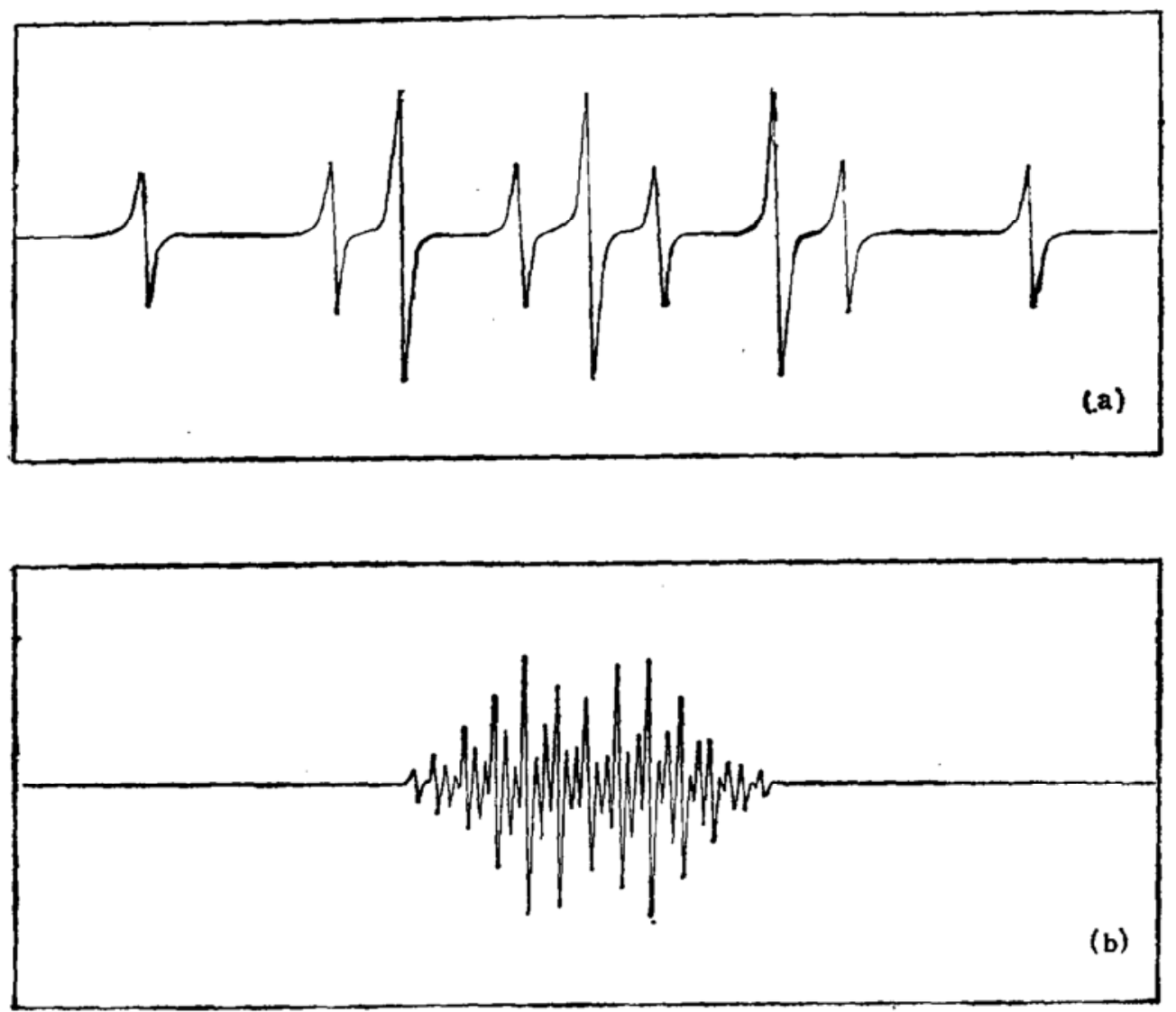

图 3

（a）对忞基苯氧自由基的模拟谱；(b) 氢原子的 DMPO 自旋加合物的模拟谱

比较图 1(a) 和图 1(b) 可知在 $\mathrm{TiO}_{2}$ 光催化对氨基酚的降解中产生了 $\mathrm{H}^{*}$ 和对氨基苯氧 自由基. 从而可确定对氨基酚的光降解是通过第一种途径 (I) 而不是通过第二种途径 (II).

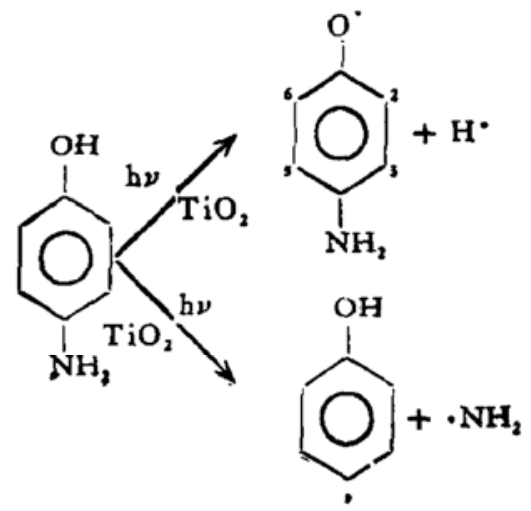


在 $\mathrm{H}_{2} \mathrm{~N}-\left\langle\bigcirc-\mathrm{O}^{\circ}\right.$ 的分裂常数中, $a_{\mathrm{NH}_{2}}^{\mathrm{N}}=0.525 \mathrm{mT}, a_{\mathrm{NH}_{2}}^{\mathrm{H}}-0.550 \mathrm{mT}$ 都较大, 即 未偶电子密度在 $\mathrm{NH}_{2}$ 基的 $\mathrm{N}$ 及 $\mathrm{H}$ 上较大, 这只有当 $\mathrm{N}$ 的孤对电子参与 $\pi$ 体系共轭才有可能. 另外, $a_{2.6}^{\mathrm{H}}-0.275 \mathrm{mT}, a_{3 . \mathrm{s}}^{\mathrm{H}}=0.175 \mathrm{mT}$, 其平均值 $a_{\mathrm{H}}=0.226 \mathrm{mT}$ 与 $-\mathrm{O}-\langle\overline{\mathrm{O}}\rangle-\mathrm{O}^{\circ}$ 中 $a_{\mathrm{H}}=0.238 \mathrm{mT}$ 相近, 表明二者相似, 都是九个电子的 $\pi$ 体系, 下面的共振结构有利其稳定.

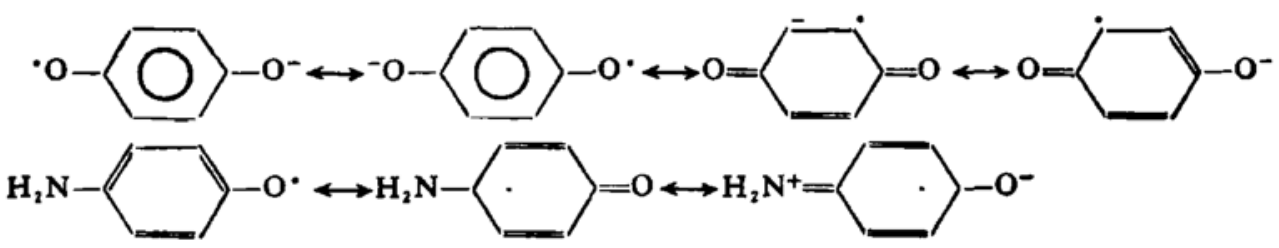

经计算可知 ${ }^{\circ} \mathrm{O}-\left\langle\mathrm{O}-\mathrm{O}^{-}\right.$及 $\mathrm{H}_{2} \mathrm{~N}-\langle\mathrm{O}\rangle-\mathrm{O}^{\circ}$ 中 $\mathrm{C}-\mathrm{O}$ 及 $\mathrm{C}-\mathrm{N}$ 的键级均为 1.5 左 右, 即有相同的强度. 在相同条件下无论是均相或非均相体系中都未观察到邻氨基酚光解产 生的

至于在非均相中 $\mathrm{H}_{2} \mathrm{~N}$ 一 $\mathrm{O}-\mathrm{O} \% /(\mathrm{DMPO}-\mathrm{H})^{\circ}$ 的比例增加约 4 倍而且分辨得好, 是 因为光照对氨基酚 $/ \mathrm{TiO}_{2}$ 的悬浮体系时, 可同时发生方程 (1),(6) 及 (7) 的反应, 所以非均相 体系中观察到 $\mathrm{H}_{2} \mathrm{~N}-\left\langle\mathrm{O}-\mathrm{O} /(\mathrm{DMPO}-\mathrm{H})^{*}\right.$ 的比例增加是不言而喻的. 此外, 多相体系 中方程(5)所示的 $\mathrm{O}_{2}$ 的消耗显然导致 ESR 谱的分辨变好.

\section{参考文 献}

[1] Kormann, C. ez al., Environ. Sci. Teahnol., 1991, 25: 494.

[2] Augugliaco, V. et al., Toxicol. Environ. Chem., 1988, (16): 89.

[3] Borello, B. et al., Environ. Toxicol. Chem., 1989, 8): 997.

[4] Hayward, N. K., Lavin, M. F., Life Sci., 1985, 36: 2039.

[ 5] Kuurata Yasushi, 名古屋私立大学医学杂誌, 1989, 40: 429.

[6] L.ipczynska-Kochqny, et al., J. Photochem. Photobiol. A: Chem., 1991, 62: 229.

[7] Neta, P., Fessenden, R. W., J. Phys. Chem., 1974, 78: 523.

[8] Ries7, P. et al., Environ. Helth Perspective, 1985, 64: 233.

[ 9 ] Sargent, F. P., Gardy, E. M., Can. J. Chem., 1976, 54: 275.

[10] Kirino, Y. et al., Chem. Pharm. Bull., 1981, 29: 29.

[11] Harbour, J. R., Hair, M. L., J. Phys. Chem., 1977, 81: 1791. 